\title{
Solvent- and catalyst-free aza-Michael addition of imidazoles and related heterocycles
}

\author{
Katharina Kodolitsch, ${ }^{a}$ Florian Gobec, ${ }^{a}$ Christian Slugovc ${ }^{a, b *}$
}

${ }^{a}$ Institute for Chemistry and Technology of Materials, Graz University of Technology, Stremayrgasse 9, A 8010 Graz, Austria; e-mail: slugovc@tugraz.at

${ }^{b}$ Christian Doppler Laboratory for Organocatalysis in Polymerization, Stremayrgasse 9, A 8010 Graz, Austria

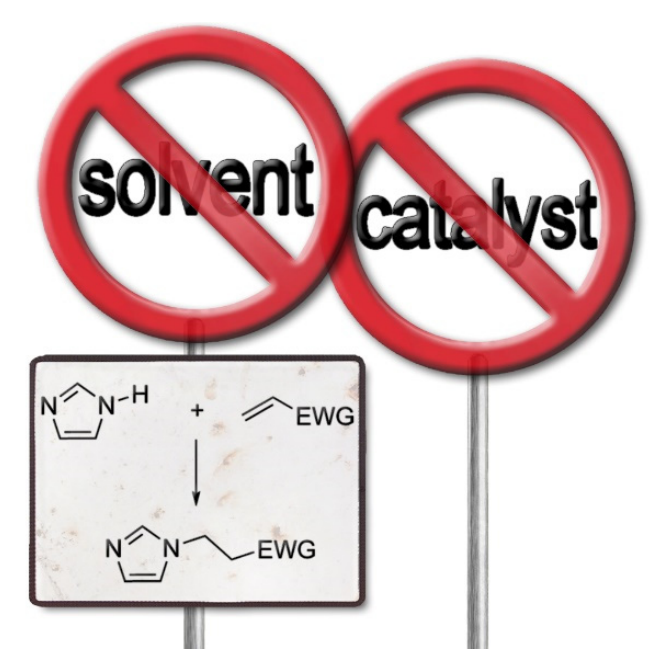

\begin{abstract}
This work demonstrates the scope and limitations of the aza-Michael addition of imidazoles and related heterocycles with electron deficient olefins under solvent- and catalyst-free conditions. The reaction proceeds at $80^{\circ} \mathrm{C}$ within hours towards completion as long as the azole derivative is sufficiently soluble in the Michael acceptor, which has been used in small excess. Workup only comprises evaporation of surplus Michael-acceptor and no additional solvents are necessary for purifying the products.
\end{abstract}




\section{Chem Rxiv ${ }^{\text {TM }}$}

Introduction. The ideal chemical reaction proceeds fast at ambient temperature and quantitatively yields a single product without the need of a catalyst. This means no side- but also no byproducts are formed, resulting in perfect atom economy ${ }^{1}$ and full consumption of educts. In this case, no purification or any other workup is necessary. In reality, the situation is in most cases different. Conversions are not complete, catalysts are needed to speed up the reaction or to provide alternative reaction pathways and/or selectivity, by- and side products form and often solvents have to be employed. Solvent use in organic chemistry is in many cases inevitable for providing high reaction rates, for obtaining the desired selectivity or for facilitating the heat management of a reaction. Moreover, many purification techniques heavily rely on the use of solvents. Accordingly, solvent use is in many cases accountable for a huge part of the synthesis' negative impact on the environment. Consequently, research striving for solvent-free and at the same time catalyst-free reactions evolved as a branch of green chemistry. ${ }^{2}$ However, many examples termed solvent-free in literature are using solvents during isolation and purification to meet the stringent requirements in terms of purity in preparative organic chemistry. Taking into account, that usually much more solvent is needed during purification than during preparation, the impact of performing a reaction without solvent (and catalyst) in saving resources is rather limited as soon as a solvent-based purification is necessary. ${ }^{3}$ In other words, solvent- and catalyst-free reactions should ideally proceed to completeness without forming side- or byproducts that have to be separated with the help of solvent-based methods in order to provide a significant advantage in terms of sustainability.

Among the many classes of different reactions, in particular addition reactions qualify for designing side- and byproduct free transformations without the need of catalysts and solvents at any stage of the preparation. Some selected examples comprise the cycloaddition of aziridines and carbon dioxide, ${ }^{4}$ hydrophosphanation of alkenes, ${ }^{5}$ Diels-Alder reactions, ${ }^{6}$ Knoevenagel reaction of aldehydes (which is a condensation type reaction) ${ }^{7}$ or the aza-Michael reactions. ${ }^{8}$ The aza-Michael reaction involves a nucleophilic amine (the Michael donor) and an electron deficient alkene (the Michael acceptor). Generally, it is performed with the aid of a catalyst under mild reaction conditions leading to high conversions with an atom economy of $100 \%$. Side-products are usually not observed. The works of Desmet et al. ${ }^{9}$ and Bláha, et al. ${ }^{8 \mathrm{c}}$ describe the mechanistic understanding of the (uncatalyzed) reaction in detail. In essence, the aza-Michael addition is a third-order reaction (first order in the acceptor and second order in the donor). The third-order 


\section{Chem Rxiv ${ }^{\text {TM }}$}

kinetics are rationalized by the formation of a rate-determining zwitterionic transition state in which a second amine is needed to facilitate the proton transfer from the attacking amine to the $\alpha$-carbon of the electron deficient olefin (Scheme 1).

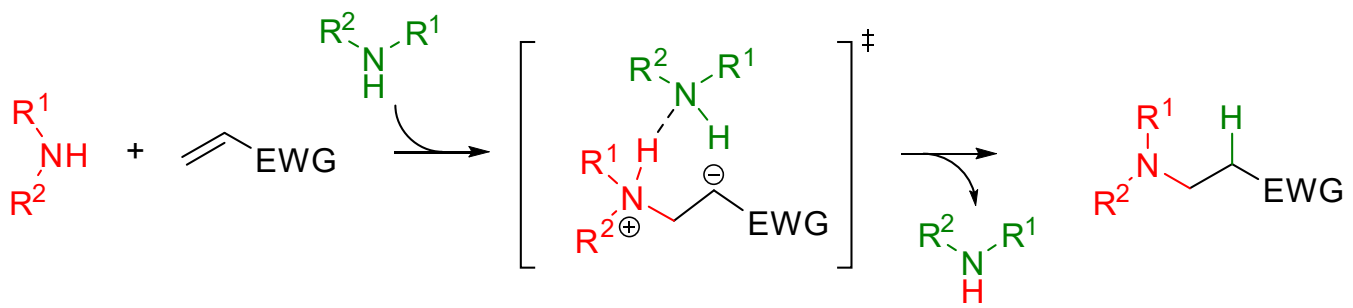

Scheme 1 Mechanism of the aza-Michael reaction according to Refs $8 \mathrm{c}$ and 9.

The mechanism implies that the aza-Michael reaction should ideally proceed with amines of high nucleophilicity (to facilitate the formation of the zwitter-ion) and bearing loosely bound protons (facilitating the hydrogen transfer). Preferably, reactions should be performed with the highest possible concentration of the reactants and not too high temperature (because the reaction is of third-order). Accordingly, the aza-Michael reaction is perfectly suitable for being performed in an uncatalyzed solvent-free manner. Surprisingly, to the best of our knowledge, only three publications on solvent- and catalyst-free aza-Michael addition are available and only aliphatic amines have been investigated so far. ${ }^{8}$

Herein, we wish to elucidate the reactivity of azoles with Michael acceptors under solvent and catalyst free conditions. Using azoles, like imidazoles or pyrazoles is an attractive target, because they show rich Michael chemistry with outreach into material, medicinal and biological chemistry. A recent review nicely summarizes the state of the art in aza-Michael additions of imidazoles. ${ }^{10}$ Interestingly it has been stated in that work, that only a few reports on reactions that satisfyingly proceed only in solvent without catalysis are known. The question whether this lack of reactivity is due to the use of unfavorable reaction conditions or the electronic features of azoles remains. The nucleophilicities and the acidities of imidazoles and related heterocycles suggest them to be poorer aza-Michael donors then aliphatic amines. Their nucleophilicity is comparable to the one of secondary aliphatic amines as judged by the methyl cation affinity (imidazole $531.7 \mathrm{~kJ} / \mathrm{mol} v s$. dimethylamine $523.1 \mathrm{~kJ} / \mathrm{mol}$ ) ${ }^{11}$ or less pronounced considering the nucleophilicity parameter $\mathrm{N}$ (imidazole $\mathrm{N}=11.47^{12} v s$. dimethylamine $\mathrm{N}=15.10^{13}$ ). Moreover, the acidity of the proton residing at the second nitrogen atom is about 3-4 orders of magnitude 


\section{Chem Rxiv ${ }^{\text {TM }}$}

lower than in aliphatic amines (e.g. imidazole $\mathrm{pK}_{\mathrm{a}}=13.9 v$ v. $\mathrm{pK}_{\mathrm{a}}=10.73$ for diethylamine).$^{14}$ The investigations presented here are intentionally restricted to Michael-acceptors, which can be separated upon evaporation in order to avoid a work-up involving any solvent.

Results and Discussion. One equivalent of the Michael donor and 1.2 equivalents of the Michael acceptor were weighted into a Schlenk tube and heated to $80^{\circ} \mathrm{C}$ under constant magnetic stirring. The reaction progress was monitored by sampling a small fraction and recording ${ }^{1} \mathrm{H}-$ NMR spectra in regular intervals. First, imidazole (1) as the Michael donor and acrylonitrile (A), methyl acrylate (B), methyl crotonate (C, methyl (E)-but-2-enoate) or methyl methacrylate (D) were used as Michael acceptors. The Michael adducts 1A and 1B quantitatively formed within 3 and $5 \mathrm{~h}$, respectively.

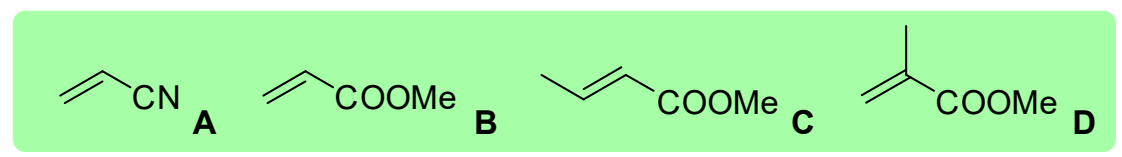

$$
\begin{array}{r}
N-H \\
\mathrm{pK}_{\mathrm{a}}=13.9
\end{array}
$$
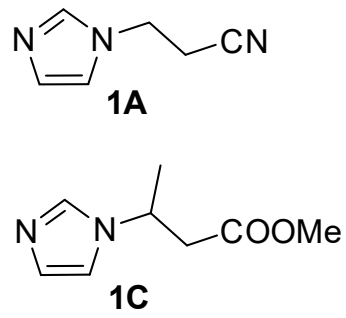
$24 \mathrm{~h}, 80^{\circ} \mathrm{C}$ $>99 \%$

$3 \mathrm{~h}, 80^{\circ} \mathrm{C}$ $>99 \%$<smiles>[B][13c]1cncn1CCC(=O)OC</smiles>

$5 \mathrm{~h}, 80^{\circ} \mathrm{C}$ $>99 \%$

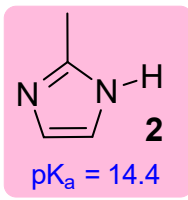<smiles>Cc1nccn1CCC#N</smiles>

2A<smiles>CC(=O)CC(C)n1ccnc1C</smiles>

$4 \mathrm{~h}, 80^{\circ} \mathrm{C}$ $>99 \%$

$24 \mathrm{~h}, 80^{\circ} \mathrm{C}$ $>99 \%$<smiles>COC(=O)C(C)Cn1ccnc1</smiles>

$24 \mathrm{~h}, 80^{\circ} \mathrm{C}$ $>99 \%$<smiles>COC(=O)CCn1ccnc1C</smiles>

2B

C $>99 \%$

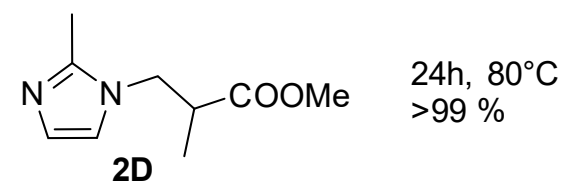

Figure 1. Conversions after given time of the aza-Michael addition of $\mathbf{1}$ and $\mathbf{2}$ to acrylonitrile (A), methyl acrylate (B), methyl (E)-but-2-enoate (C) and methyl methacrylate (D) performed at $80^{\circ} \mathrm{C}$ under solvent and catalyst free conditions. Isolated yields are in all cases above $97 \%$.

When using $\mathbf{C}$ and $\mathbf{D}$ as the Michael acceptors complete conversion was observed after about 24

h. The different speeds of the reaction can be attributed to the different electrophilicity 


\section{Chem Rxiv ${ }^{\text {TM }}$}

(expressed by the electrophilicity parameter E) of the employed Michael acceptors decreasing in the order of $\mathbf{B}(-18.84) \approx \mathbf{A}(-19.05)>>\mathbf{D}(-22.27) \approx \mathbf{C}$ (approx. -23$).{ }^{15}$ The reaction of 2-methyl imidazole (2) with A-D was somewhat slower than with 1. This might be explained by the lower $\mathrm{pK}_{\mathrm{a}}$-value calculated for $\mathbf{2}$ when compared to $\mathbf{1}$ (Fig. 1), since the nucleophilicity of $\mathbf{2}$ is slightly higher $\left(\mathrm{N}=11.74\right.$ vs. $\left.\mathbf{1}: \mathrm{N}=11.47^{12}\right)$.

To further examine the impact of the acidity of the heterocycles' protons, a selection of different Michel donors covering a range of different acidities was made (Fig. 2, left). Their performance in the reaction with methyl acrylate $(\mathbf{B})$ was investigated under the same reaction conditions as used before. Relatively acidic 1,2,4-triazole (3, exhibiting a $\mathrm{pK}_{\mathrm{a}}$ of 10.2) resulted in quantitative conversion in only $2 \mathrm{~h}$ (amongst $3 \mathrm{~B}$ also a second unidentified product formed to about 10\%). In contrast, 2-phenylimidazole (4) needed 23 times longer. Compound 4 is distinctly less acidic than $\mathbf{3}$ but more acidic than $\mathbf{1}$ and $\mathbf{2}$. Similarly, benzo[d]imidazole $\left(\mathbf{5}, \mathrm{pK}_{\mathrm{a}}=13.9 ; \mathrm{N}=\right.$ 10.50 ) needs $49 \mathrm{~h}$ for being fully converted into 5B. In these two cases, the solubility of the heterocycle in $\mathbf{B}$ is low. While in all other cases discussed so far, the Michael donor compound dissolved within the first 5-15 min of the reaction time in the acceptor component, $\mathbf{4}$ and $\mathbf{5}$ needed hours to dissolve completely. Increasing the amount of $\mathbf{B}$ to $3.7 \mathrm{eq}$. in respect to $\mathbf{5}$ allowed for obtaining full conversion after $5.5 \mathrm{~h}$. Accordingly, the poor solubility of $\mathbf{5}$ in $\mathbf{B}$ (and analogously of $\mathbf{4}$ in $\mathbf{B}$ ) is governing the speed of the reaction. Pyrazole (6) featuring a pKa value of 14.0 dissolves fast and gives $\mathbf{6 B}$ in full conversion after 4 h, i.e. 6 is converted at a similar rate like 1 and 2. Finally, in the case of pyrrole $\left(7, \mathrm{pK}_{\mathrm{a}}=17.0\right)$ no reaction with methyl acrylate under the given conditions was observed. Since $\mathbf{7}$ is a liquid which is miscible with $\mathbf{B}$, the unsuccessful conversion towards 7B has to be attributed to its electronic properties. Compound $\mathbf{7}$ is a poorer nucleophile $\left(\mathrm{N}=4.63^{16}\right)$ than all the other heterocycles under investigation and additionally proton transfer is inhibited because of its low acidity (which is even lower than that of alcohols ${ }^{17}$ ). Imidazole based heterocycles like 2-methyl-4-nitro-1H-imidazole, adenine or 1,9dimethylguanine are insoluble in $\mathbf{B}$ and showed no conversion towards the respective Michael addition product within $24 \mathrm{~h}$.

Switching from Michael acceptor $\mathbf{B}$ to the less electrophilic $\left(E=-20.22^{15}\right)$ and less polar $t$-butyl acrylate (E) corroborates the influence of solubility on the speed of the reaction. Conversions with the azoles 1-6 after about $24 \mathrm{~h}$ are not complete and numbers are given in Fig. 2 (right). In 


\section{Chem Rxiv ${ }^{T M}$}

all cases, heterocycles dissolved more slowly contributing to the distinctly longer reaction times. This was confirmed by using a higher excess of $\mathbf{E}$ ( 5 equiv.) which allowed for shortening the reaction time giving a conversion of $97 \%$ towards $2 \mathbf{E}$ in $24 \mathrm{~h}$. Accordingly, in these cases, a solvent and catalyst free protocol is lacking merit.

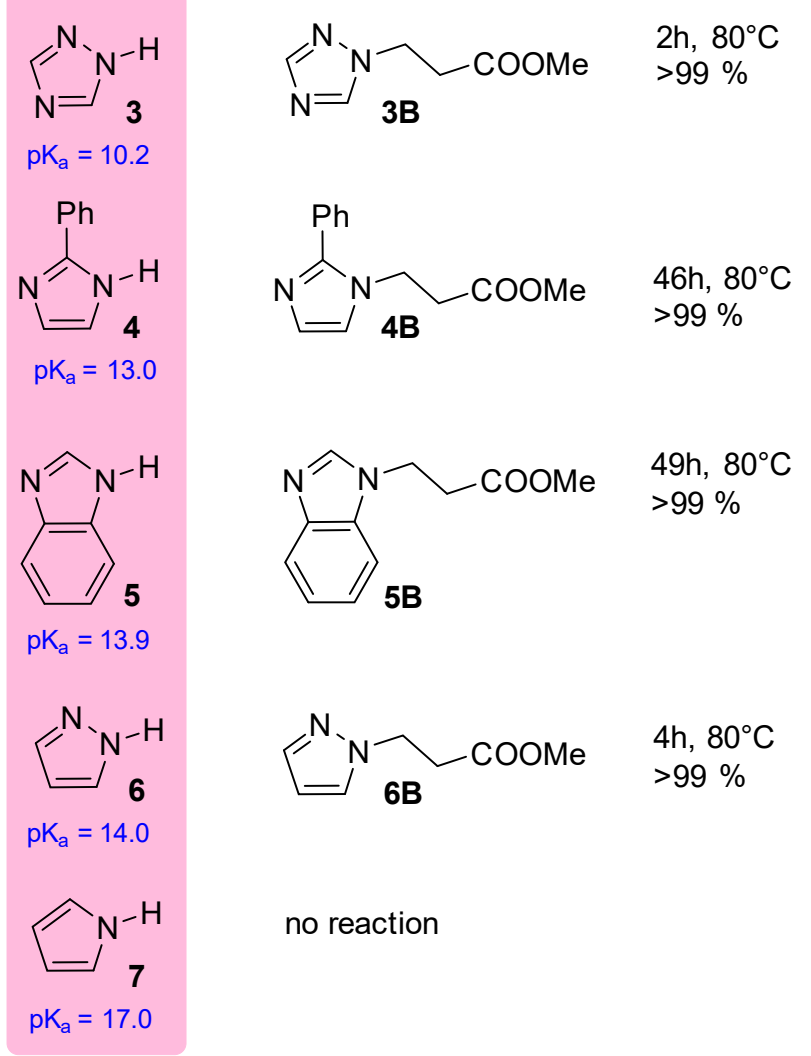

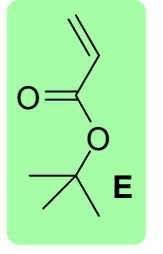

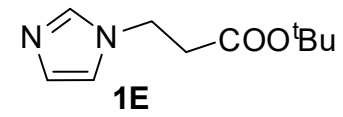

$24 \mathrm{~h}, 80^{\circ} \mathrm{C}$ $80 \%$<smiles>CCOC(=O)CCn1ccnc1C</smiles>
$26 \mathrm{~h}, 80^{\circ} \mathrm{C}$ $75 \%$<smiles>CCCOC(=O)CCn1cncn1</smiles>
$21 \mathrm{~h}, 80^{\circ} \mathrm{C}$ $70 \%$<smiles>CCOC(=O)CCn1ccnc1-c1ccccc1</smiles>
$24 \mathrm{~h}, 80^{\circ} \mathrm{C}$ $26 \%$<smiles>CCCCOC(=O)CCn1cnc2ccccc21</smiles>
$24 \mathrm{~h}, 80^{\circ} \mathrm{C}$ $10 \%$<smiles>CCCCOC(=O)CCn1cccn1</smiles>

Figure 2. Conversions after given time of the aza-Michael addition of 3-7 with methyl acrylate (B) or $t$ butyl acrylate $(\mathbf{E})$ performed at $80^{\circ} \mathrm{C}$ under solvent and catalyst free conditions. Isolated yields of 3B-6B are above $97 \%$.

In a next step, the preparation was further simplified and stirring was omitted in order not to lose yield when removing the magnetic stirring bar. The Michael-donor (1 eq.) and the Michael acceptor (1.1 eq.) were combined in a flat bottom tube, the tube was locked and placed in an oven operated at $80^{\circ} \mathrm{C}$. Under these conditions, the reactions needed two to three times longer to reach completeness. Checking the conversion via repeated ${ }^{1} \mathrm{H}-\mathrm{NMR}$ measurements of the reaction of 2 with $\mathbf{B}$ revealed a fast reaction (half-life $<6 \mathrm{~min} ; 93 \%$ conversion at $216 \mathrm{~min}$ ) 


\section{Chem Rxiv ${ }^{\text {TM }}$}

which only sluggishly reached completion (at about $18 \mathrm{~h}$ ). The excess of $\mathbf{B}$ was then removed under vacuum. It is worth noting, that also under these conditions, no side product or decomposition products were observed.

Comparing the obtained results with some from catalytic studies of the aza-Michael addition illustrates the importance of using as little solvent as possible for obtaining fast and quantitative conversion. In a first example from literature, the preparation of compound $\mathbf{1 B}$ using $\mathrm{I}_{2}$ as the catalyst in refluxing toluene (concentration of $\mathbf{1}=1 \mathrm{~mol} / \mathrm{L}$ ) was described. ${ }^{18}$ The reaction reached completion after $9 \mathrm{~h}$ and a yield of 50\% upon work-up was obtained. Another example reported that the reaction of $\mathbf{1}(\mathrm{c}=0.25 \mathrm{~mol} / \mathrm{L})$ and $\mathbf{B}$ catalyzed by alkaline protease from Bacillus subtilis at $50{ }^{\circ} \mathrm{C}$ in pyridine needed a reaction time of $72 \mathrm{~h}$ and yielded $76 \%$ of $1 \mathbf{B} .{ }^{19} \mathrm{In}$ a final example of the many protocols available in literature, ${ }^{10} \mathbf{1}(\mathrm{c}=2 \mathrm{~mol} / \mathrm{L})$ and $\mathbf{B}$ were reacted in acetonitrile at room temperature using 0.5 equiv. of 1,8-diazabicyclo[5.4.0]undec-7ene (DBU) as catalyst. Tha aza-Michael adduct 1B was obtained in $95 \%$ yield after 14 h. $^{20}$ Recalling the performance of the protocol disclosed herein (solvent and catalyst free, $5 \mathrm{~h}$ at $80^{\circ} \mathrm{C},>97 \%$ yield) clearly reveals that aza-Michael reactions should be carried out in solution using the highest possible concentration of the reactants.

Conclusions. In summary, it has been shown that azoles and related compounds undergo solvent- and catalyst-free Michael addition with methyl acrylates and acrylonitrile in reasonable time as long as the Michael donor is sufficiently soluble in the Michael acceptor at $80^{\circ} \mathrm{C}$. Workup only comprises the removal of the small excess of the Michael acceptor by evaporation. Weaker Michael acceptors being at the same time poorer solvents for the azole derivatives require reaction times of more than $24 \mathrm{~h}$ to reach completeness. In such cases, the use of solvents and catalysts is advisable. The findings contain a caveat for all researching catalysis in azaMichael additions, namely that the concentration of the reaction partners is of crucial importance for determining the speed of the reaction, just as expected for a third-order reaction. Finally, the statement issued in a recent review, that only few reports on reactions that satisfyingly proceed only in solvent without catalysis are known, has been demystified. 


\section{Chem Rxivin}

Experimental. All reactions were performed under ambient conditions. Chemicals were purchased from TCI, Acros or Sigma-Aldrich and were used as received (stabilizers present in the Michael acceptors were not removed). ${ }^{1} \mathrm{H}$ and ${ }^{13} \mathrm{C}$-NMR spectra were recorded at $23{ }^{\circ} \mathrm{C}$ on a Bruker Ultrashield 300 spectrometer operating at $300.36 \mathrm{MHz}\left({ }^{1} \mathrm{H}\right)$ and $75.53 \mathrm{MHz}\left({ }^{13} \mathrm{C}\right)$. Deuterated solvents were purchased from Cambridge Isotope Laboratories.

A general procedure is given by the example of the preparation of 2B. Methyl acrylate (3.3 mL, $0.0364 \mathrm{~mol}, 1.2$ eq.) was added to 2 -methylimidazole $(2.487 \mathrm{~g}, 0.0303 \mathrm{~mol}, 1.0$ eq.) in a $15 \mathrm{~mL}$ Schlenk tube. The suspension was heated up to $80{ }^{\circ} \mathrm{C}$ under constant stirring whereupon dissolution of 2-methylimidazole occurred within approx. $5 \mathrm{~min}$. After $6 \mathrm{~h}$ the reaction mixture was cooled to room temperature and the resulting oil was dried under reduced pressure (to get rid of the excess of methyl acrylate) whereupon the colorless oil solidified. The magnetic stirrer bar was removed from the product. Yield: $4.97 \mathrm{~g},(0.0295 \mathrm{~mol}), 97.5 \% \mathrm{o}$. Th., colorless powder. Analytical data in accordance with literature. ${ }^{21}$

Supporting Information available: Analytical data of the products

Acknowledgment. Financial support via the Christian Doppler Society ("CD-Laboratory for Organocatalysis in Polymerization") and Graz University of Technology (LP-03 "Porous Materials@Work") is gratefully acknowledged.

\section{References}

1 The atom economy-a search for synthetic efficiency. Trost, B. M. Science 1991, 254, 1471-1477. DOI: $\underline{10.1126 / \text { science. } 1962206}$

2 a) Solvent-Free and Catalysts-Free Chemistry: A Benign Pathway to Sustainability. Gawande, M. B.; Bonifucio, V. D. B.; Luque, R.; Branco, B. S.; Varma, R. S. ChemSusChem 2014, 7 ,24-44. DOI: 10.1002/cssc.201300485; b) A decade update on solvent and catalyst-free neat organic reactions: a step forward towards sustainability. Sarkar, A.; Santra, S.; Kundu, S. K.; Hajra, A.; Zyryanov, G. V.; Chupakhin, O. N.; Charushin, V. N.; Majee, A. Green Chem. 2016, 18, 4475-4525. DOI: 10.1039/C6GC01279E

3 Toward the ideal synthesis and molecular function through synthesis-informed design. Wender, P.A. Nat. Prod. Rep. 2014, 31, 433-440. DOI: 10.1039/C4NP00013G

4 a) Catalyst-Free Process for the Synthesis of 5-Aryl-2-Oxazolidinones via Cycloaddition Reaction of Aziridines and Carbon Dioxide. Dou, X.-Y.; He, L.-N.; Yang, Z.-Z.; Wang, J.-L. Synlett 2010, 14, 2159-2163. DOI: $\underline{10.1055 / \mathrm{s}-0030-1258510}$; b) The solvent-free and catalyst-free conversion of an aziridine to an oxazolidinone using only carbon dioxide. Phung, C.; Ulrich, R. M.; Ibrahim, M.; Tighe, N. T. G.; Lieberman, D. L.; Pinhas, A. R. Green Chem. 2011, 13, 3224-3229. DOI: 10.1039/C1GC15850C

5 a) Solvent- and catalyst-free regioselective hydrophosphanation of alkenes. Alonso, F.; Moglie, Y.; Radivoy, G.; Yus, M. Green Chem. 2012, 14, 2699-2702. DOI: 10.1039/C2GC35898K; b) Catalyst- and solvent-free 
hydrophosphination and multicomponent hydrothiophosphination of alkenes and alkynes. Moglie, Y.; GonzálezSoria, M. J.; Martín-García, I.; Radivoy, G.; Alonso, F. Green Chem. 2016, 18, 4896-4907. DOI: 10.1039/C6GC00903D

6 a) Solvent-free Diels-Alder reactions of in situ generated cyclopentadiene. Huertas, D.; Florscher, M.; Dragojlovic, V. Green Chem. 2009, 11, 91-95. DOI: 10.1039/B813485E; b) Converting natural rubber waste into Ring-opening Metathesis polymers with oligo-1,4-cis-isoprene sidechains. Abbas, M.; Neubauer, M.; Slugovc, C. Polym. Chem. 2018, 9, 1763-1766. DOI: 10.1039/C8PY00233A

7 Catalyst-free and Solvent-free Cyanosilylation and Knoevenagel Condensation of Aldehydes. Wang, W.; Luo, M.; Yao, W.; Ma, M.; Pullarkat, S. A.; Xu, L.; Leung, P.-H. ACS Sustainable Chem. Eng. 2019, 7, 1718-1722. DOI: $\underline{10.1021 / \text { acssuschemeng.8b05486 }}$

8 a) Solvent-free, catalyst-free Michael-type addition of amines to electron-deficient alkenes. Ranu, B. C.; Dey, S. S.; Hajra, A. Arkivoc 2002, 7, 76-81. DOI: 10.3998/ark.5550190.0003.709; b) Uncatalyzed, green aza-Michael addition of amines to dimethyl maleate. Bosica, G.; Debono, A. J. Tetrahedron 2014, 70, 6607-6612. DOI: 10.1016/j.tet.2014.06.124; Solvent-free, catalyst-free aza-Michael addition of cyclohexylamine to diethyl maleate: Reaction mechanism and kinetics. Bláha, M.; Trhlíková, O.; Podešva, J.; Abbrent, S.; Steinhart, M.; Dybal, J.; Dušková-Smrčková, M. Tetrahedron 2018, 74, 58-67. DOI: 10.1016/j.tet.2017.11.033

9 Quantitative First-Principles Kinetic Modeling of the Aza-Michael Addition to Acrylates in Polar Aprotic Solvents. Desmet, G. B.; D’hooge, D. R.; Omurtag, P. S.; Espeel, P.; Marin, G. P.; Du Prez, F. E.; Reyniers, M.F. J. Org. Chem. 2016, 81, 12291-12302. DOI: 10.1021/acs.joc.6b02218

10 Aza-Michael Addition of Imidazole Analogues. Gmach, J.; Joachimiak, Ł.; Błażewska, K. M. Synthesis 2016, 48, 2681-2704. DOI: $10.1055 / \mathrm{s}-0035-1560451$

11 a) Cation affinity numbers of Lewis bases. Lindner, C.; Tandon, R.; Maryasin, B.; Larionov, E.; Zipse, H. Beilstein J. Org. Chem. 2012, 8, 1406-1442. DOI:10.3762/bjoc.8.163; b) Methyl Cation Affinities of Commonly Used Organocatalysts. Wie, Y.; Sastry, G. N.; Zipse, H. J. Am. Chem. Soc. 2008, 130, 3473-3477. DOI: $10.1021 /$ ja0764409

12 Nucleophilicities and Lewis basicities of imidazoles, benzimidazoles, and benzotriazoles. Baidya, M.; Brotzel, F.; Mayr, H. Org. Biomol. Chem. 2010, 8, 1929-1935. DOI: 10.1039/C000965B

13 Nucleophilic Reactivities of Primary and Secondary Amines in Acetonitrile. Kanzian, T.; Nigst, T. A.; Maier, A.; Pichl, S.; Mayr, H. Eur. J. Org. Chem. 2009, 6379-6385. DOI: 10.1002/ejoc.200900925

14 values from Advanced Chemistry Development (ACD/Labs) Software V11.02, retrieved from SciFinder.

15 Quantification and Theoretical Analysis of the Electrophilicities of Michael Acceptors. Allgäuer, D. S.; Jangra, H.; Asahara, H.; Li, Z.; Chen, Q.; Zipse, H.; Ofial, A. R.; Mayr, H. J. Am. Chem. Soc. 2017, 139, 13318-13329. DOI: $10.1021 /$ jacs.7b05106

16 Structure - Nucleophilicity Relationships for Enamines. Kempf, B.; Hampel, N.; Ofial, A. R.; Mayr, H. Chem. Eur. J. 2003, 9, 2209-2218. DOI: 10.1002/chem.200204666

17 a) Nucleophile-mediated oxa-Michael addition reactions of divinyl sulfone - a thiol-free option for step-growth polymerisations. Strasser, S.; Slugovc, C. Catal. Sci. Technol. 2015, 5, 5091-5094. DOI: 10.1039/c5cy01527h ; b) Solvent-free macrocyclisation by nucleophile-mediated oxa-Michael addition polymerisation of divinyl sulfone and alcohols. Strasser, S.; Wappl. C.; Slugovc. C. Polym. Chem. 2017, 8, 1797-1804. DOI: 10.1039/C7PY00152E

18 Aza-Michael Addition of Amines to $\alpha, \beta$-Unsaturated Compounds Using Molecular Iodine as Catalyst. Borah, K. J.; Phukan, M.; Borah, R. Synth. Commun. 2010, 40, 2830-2836. DOI: 10.1080/00397910903320241

19 Hydrolase-catalyzed Michael addition of imidazoles to acrylic monomers in organic medium. Cai, Y.; Wu, Q.; Xiao, Y.; Lv, D.; Lin, X. J. Biotechnol. 2006, 121, 330-337. DOI: 10.1016/j.jbiotec.2005.07.001

20 1,8-Diazabicyclo[5.4.0] undec-7-ene (DBU)-promoted efficient and versatile aza-Michael addition. Yeom, C.-E.; Kim, M. J.; Kim, B. M. Tetrahedron 2007, 63, 904-909. DOI: 10.1016/j.tet.2006.11.037

21 One-Pot Consecutive Catalysis by Integrating Organometallic Catalysis with Organocatalysis. Sau, S. C.; Roy, S. R.; Mandal, S. K. Chem. Asian J. 2014, 9, 2806-2813. DOI: 10.1002/asia.201402363 